\title{
Pre-pectoral breast reconstruction: early and long-term safety evaluation of 146 unselected cases of the early pre-pectoral era of a single-institution, including cases with previous breast irradiation and post-mastectomy radiation therapy
}

\author{
Marco Bernini $^{1}$ (D) Icro Meattini ${ }^{2,3} \cdot$ Calogero Saieva $^{4} \cdot$ Carlotta Becherini $^{2} \cdot$ Viola Salvestrini $^{2} \cdot$ Luca Visani $^{2}$. \\ Giulia Stocchi ${ }^{2}$. Chiara Bellini ${ }^{2}$. Victoria Lorenzetti ${ }^{2}$. Silvia Sordi ${ }^{1}$. Jacopo Nori ${ }^{5}$. Diego De Benedetto ${ }^{5}$. \\ Isacco Desideri $^{2,3} \cdot$ Simonetta Bianchi ${ }^{6} \cdot$ Lorenzo Livi $^{2,3} \cdot$ Lorenzo Orzalesi $^{1}$
}

Received: 3 April 2021 / Accepted: 31 October 2021

(c) The Author(s), under exclusive licence to The Japanese Breast Cancer Society 2021

\begin{abstract}
We re-evaluated acute and early-late toxicity-related factors among pre-pectoral immediate tissue expander/implant (TE/I) breast reconstruction (BR) unselected, first-era, cases, including previous breast radiation treatment and post-mastectomy radiation therapy (PMRT). A retrospective analysis of 146 (117 therapeutic and 29 prophylactic) pre-pectoral reconstructions, between 2012 and 2016, considered patient-related (age, body mass index [BMI], smoke-history, comorbidity, BRCA mutation), and treatment-related characteristics (previous irradiation, axillary surgery, PMRT, pre- and postoperative chemotherapy, endocrine therapy, and target-therapy). Safety was evaluated as acute and early-late complications, and TE/I failures. At multivariate analysis of the 146 cases (117 patients submitted to BR) a significant factor related to acute toxicity was: $\mathrm{BMI} \geq 25$ (31.3\% [ $\geq 25]$ vs $8.8 \%$ [<25]; OR 4.44, 95\% CI 1.56-12.6; $p=0.003)$, while previous breast surgery on ipsilateral side presented a borderline significance (31.6\% [previous surgery] vs $7.4 \%$ [no previous surgery]; OR $3.74,95 \%$ CI $0.97-14.40 ; p=0.055)$. Factors significantly related to TE/I failure were: current or previous smoking exposition (13.8\% [smokers] vs $2.6 \%$ [non-smokers]; OR 7.32, 95\% CI 1.37-39.08; $p=0.02)$ and preoperative chemotherapy (18.8\% [yes] vs $3.5 \%$ [no]; OR 8.16, 95\% CI 1.29-51.63; $p=0.026$ ). At 4-year median follow-up, 3 deaths, 5 locoregional recurrences, and 14 distant metastases occurred. Immediate pre-pectoral BR is safe and effective, with low rates of acute and early-late complications. BMI and previous breast surgery were related to higher complications but not failure; smoking and preoperative chemotherapy were related to TE/I explant. Previous RT and PMRT were related neither to early-late toxicity nor failure.
\end{abstract}

Keywords Pre-pectoral breast reconstruction · Post-mastectomy radiation therapy $\cdot$ Implant based breast reconstruction

Marco Bernini

marco.bern@tin.it

1 Breast Surgery, Breast Unit, Oncology Department, Azienda Ospedaliero Universitaria Careggi, University of Florence, L.go Brambilla 3, 50134 Florence, Italy

2 Department of Biomedical, Experimental and Clinical Sciences "M. Serio", University of Florence, Florence, Italy

3 Radiotherapy Unit, Oncology Department, Azienda Ospedaliero Universitaria Careggi, Florence, Italy

4 Istituto per lo Studio, la Prevenzione e la Rete Oncologica (ISPRO), Florence, Italy

5 Diagnostic Senology, Azienda Ospedaliero Universitaria Careggi, University of Florence, Florence, Italy

6 Pathology Division, Azienda Ospedaliero Universitaria Careggi, University of Florence, Florence, Italy

\section{Introduction}

Implant-based breast reconstruction (IBBR), either one- or two-stage by means of tissue expander (TE), is nowadays the most frequent reconstructive choice particularly in conservative mastectomies [1, 2]. After initial diffusion of subcutaneous implant and expander placement in the early era of prosthetic reconstruction [3-5], the development of modified radical mastectomy, e.g. saving the pectoralis major muscle, allowed the introduction of retro-pectoral implant reconstruction, which largely replaced the subcutaneous approach, due to a significant lower rate of postsurgical complications, such as prosthesis extrusion and capsular contracture $[6,7]$. 
The introduction of biological or synthetic soft tissue replacement devices allowed the so called direct-to-implant (DTI) one-step IBBR with a dual plane retro-pectoral technique, using matrixes as a hammock in the lower pole [8]. Later on, because of the good results of soft tissue replacement devices in a dual plane approach, the rationale in favor of using such devices as a full coverage of TE/I was developed and published first by means of synthetic meshes [9], and immediately after by acellular dermal matrixes (ADMs) [10], thus starting again the era of a subcutaneous approach, this time considered a conservative reconstruction with a complete muscle sparing technique. More recently, many experiences and series have been published in literature reporting the growing popularity of this novel approach in BR [11-25]. And nowadays several algorithms and selection criteria have been proposed to identify the candidates of this new, very popular, approach [26].

The aim of the present study is to go back to the very beginning of the pre-pectoral (pre-pec) era, where patients were mostly unselected, to evaluate the acute and early-late toxicity predictive factors and safety in patients receiving pre-pectoral immediate TE/I based reconstruction. Such an analysis was motivated by the intent to analyze the risk of pre-pec IBBRs in relation to radiation therapy, performed either before or after the reconstruction itself, which is a hot issue in the present era of the pre-pec IBBRs. All the preoperative characteristics and also the post-operative treatments, such as postmastectomy radiation therapy (PMRT), were analyzed as risk factors for complication and failures in an early group of patients of a single Institution, which was one of the first to adopt such an approach. Going back to a very early age of this experience, when selection criteria were quite loose, allowed to assess if RT is a possible risk factor, with a multivariate analysis that considered many other features.

\section{Patients and methods}

\section{Patients}

We performed a retrospective analysis of 117 consecutive patients who underwent therapeutic or prophylactic pre-pectoral IBBR from October 2012 to May 2016 at our Center (117 therapeutic and 29 prophylactic). Patients underwent DTI pre-pectoral reconstructions or two-stage TE pre-pectoral implant-based BR.

We recorded individual patient-related features (i.e., age, body mass index [BMI], smoke-history, comorbidity, BRCA mutation, previous RT), and BC-related treatments characteristics (i.e., axillary surgery, adjuvant post-mastectomy radiotherapy [PMRT], preoperative and postoperative chemotherapy, endocrine therapy, and target therapy).
Toxicity profile was evaluated in terms of complications related to IBBR; we recorded acute toxicity, late toxicity, and TE/I explantation rate, considered as a failure. As previously published $[9,12,13]$ acute toxicities were classified as follows: BR failure (i.e., TE/I explantation) and surgical complications, namely, skin-nipple necrosis, seroma, wound dehiscence, surgical site infection, hematoma, and atopic versus graft reaction. Early-late toxicities (assessed at 2 years from the DTI reconstruction, and at least after one year form second stage in every TE case) were classified as follows: chronic seroma, infection, capsular contracture, extrusion/damage of the implant.

\section{Treatments}

Surgical techniques for DTI [9] and for two-stage TE reconstructions have been previously described [13]. Patients scheduled at our institution for conservative mastectomies, either nipple-sparing or skin-sparing mastectomy, were thoroughly informed of different reconstruction options, either autologous or prosthetic. The pre-pec approach was thoroughly described and an informed consent was signed by every patient. The pre-pectoral technique was started at our institution in 2011 as a pilot protocol approved by the Hospital Drugs and Devices Service Committee, designed in accordance with the Hospital Ethical Committee rules on non-randomized clinical studies, and it was conducted in accordance with the ethical standards laid down in the 1964 Declaration of Helsinki and its later amendments.

If a TE approach was chosen, patients were informed of the muscle-sparing subcutaneous option with synthetic mesh coverage only (totally subcutaneous, pre-pectoral TE adjustment, and wrapped in a titanium-coated polypropylene synthetic mesh bag, TiLOOP ${ }^{\circledR}$ Bra, pfm medical, Cologne Germany). In case of two-stage IBBR mesh wrapping around TE was loose, considering final expansion diameter of TE. Outpatient expansions were done every week or every 2 weeks for the first 2 months, with $40-50 \mathrm{~mL}$ of sterile solution each time [13]. On the other hand, in case of DTI the synthetic mesh wrapping was tight around the chosen implant adjusting the mesh itself by means of a purse string suture [12]. A specific digital database was adopted in 2012 to prospectively collect all the consecutive pre-pectoral cases performed, encompassing all baseline characteristics, oncological parameters, therapies and follow-up, surgical complications, outpatient visits, re-interventions, second stage reconstructions in case of TE, and long-term outcomes. All further surgical procedures and postoperative radiation therapies, occurred between the first reconstruction and last follow-up, were investigated and registered.

All patients with a 2-year minimum follow-up were submitted to both an objective and a subjective long-term outcomes evaluation. At the objective long-term evaluation a 
score was given to the capsular contracture according the 4-grade Baker scale [27]. TE reconstruction cases were all at 1 year from second-stage by the time of the 2-year long-term evaluation. PMRT (when indicated) was always performed between first and second surgical stage. The subjective evaluation was conducted using the postoperative section of BREAST-Q reconstruction module (Memorial Sloan-Kettering Cancer Center and The University of British Columbia ${ }^{\odot} 2006$, all rights reserved). Our experiences in subjective assessment was previously published and did not represent the aim of the present study [13].

All patients were discussed case-by-case by our breast cancer multidisciplinary team. Systemic therapies indications, included preoperative chemotherapy and postoperative chemotherapy/endocrine therapy, followed national and international oncological guidelines. When indicated, PMRT volumes and doses consisted of affected chest-wall plus ipsilateral axillary 3-4 level irradiation (50 Gy in 25 fractions), independently of axillary surgical treatment. Axillary 1-2 levels and internal mammary nodes were never irradiated in present series, since at our Institution the radiation treatment volumes include not operated axillary levels without internal mammary nodes. Internal mammary nodes are included only if positive or suspicious at diagnostic imaging. PMRT included the definitive implant or tissue expander and was delivered between 10 and 20 weeks from surgery (in any case before the second-stage reconstruction); in case of postoperative chemotherapy, PMRT was delivered at the end of treatment (between 4 and 6 weeks from the last cycle). Conversely, PMRT was given concomitant to adjuvant endocrine therapy (in case of both aromatase inhibitors and tamoxifen).

\section{Pathology methods}

Every case was diagnosed by specialized pathologists, dedicated to breast diseases. Diagnosis was made according to the AJCC and WHO criteria. Starting from macroscopic examination, in order to correctly diagnose possible site/s of microinvasion, handling of surgical specimens included "bread-slicing" method: mastectomies specimens were serially sectioned at $1.5-2 \mathrm{~mm}$ intervals from superficial to deep aspects. Tissue sections were chosen for microscopic examination on corresponding mammographic patterns in case of mastectomy. Focus or foci of microcalcifications were completely and sequentially submitted to histological examination. Cancer subtype (Luminal A, Luminal B HER-2 negative, Luminal B HER-2 positive, HER-2 positive and Triple Negative) was determined by ER, PR receptors, and HER-2 status. Ki-67 stain was used for distinction of Luminal A and B. Hormone receptor status was reported as negative when $<1 \%$ of tumors cells stained at IHC. HER2 status was determined only by IHC in cases scored as 0 or
$1+$ (negative) and $3+$ (positive), otherwise a FISH test was adopted.

\section{Statistical analysis}

The association between selected individual characteristics and selected toxicities was firstly evaluated by a simple chisquare test (Table 1). Secondly, a univariate logistic analysis was performed to estimate the association between each individual parameter and selected toxicities (Tables 2 and 3). The risk of toxicity was calculated by odd ratios (OR) and 95\% confidence intervals $(95 \% \mathrm{CI})$. A logistic multivariate analysis including parameters resulted to be statistically significant at univariate analysis was finally performed.

Survival analyses were performed in relation to specific events: local recurrences (LR), distant metastasis (DM), and death. Overall survival (OS) was defined as the time from the surgery to time of death or last follow-up. Progressionfree survival (PFS) as time to specific events (LR or DM) was measured from the date of surgery to the date of event. Patients who died before experiencing a disease occurrence were considered censored at their dates of death. Event rates and their $95 \% \mathrm{CI}$ were calculated according to the Kaplan-Meier method.

Differences between groups of patients were evaluated using the log-rank test. Univariate Cox proportional regression model was used to obtain the hazard ratios (HR) and corresponding $95 \% \mathrm{CI}$ for specific events. A multivariate Cox proportional regression model was used to identify independent factors of specific events. All two-sided p-values less than 0.05 were considered significant. Statistical analyses were performed using SPSS Statistics software (version 22; SPSS Statistics, IBM Corporation, Armonk, NY, USA).

\section{Results}

\section{Patients' characteristics and surgical complication rates}

We analyzed 117 patients, 88 had a unilateral mastectomy, while 29 had a bilateral mastectomy, with an overall number of 146 consecutive pre-pectoral IBBRs, 117 therapeutic and 29 prophylactic mastectomies. A bilateral procedure was performed in 6 patients with bilateral cancer, in 6 patients with a BRCA mutation and in 17 women who chose to remove the contralateral breast. The 12 bilateral mastectomies in 6 BRCA-mutation carriers and the 17 contralateral mastectomies represented the 29 cases of prophylactic mastectomies (until recently, a prophylactic mastectomy was offered, at our Institution, both to BRCA-mutation carries and to those women scheduled 
Table 1 Main features of the whole series

\begin{tabular}{|c|c|c|c|c|}
\hline & $\begin{array}{l}\text { Cases } \\
n\end{array}$ & $\begin{array}{l}\text { Acute toxicity } \\
n(\%)\end{array}$ & $\begin{array}{l}\text { TE/I explant } \\
n(\%)\end{array}$ & $\begin{array}{l}\text { Late toxicity } \\
n(\%)\end{array}$ \\
\hline \multicolumn{5}{|l|}{ Group age, years } \\
\hline$<45$ & 35 & $5(14.3)$ & $2(5.7)$ & 0 \\
\hline $46-55$ & 64 & $7(10.9)$ & $2(3.1)$ & $2(3.1)$ \\
\hline$>55$ & 47 & $8(17.0)$ & $3(6.4)$ & 0 \\
\hline$p$ value & & 0.65 & 0.70 & 0.27 \\
\hline \multicolumn{5}{|l|}{ Smoking habits } \\
\hline Never smoker & 117 & $15(12.8)$ & $3(2.6)$ & $1(0.8)$ \\
\hline Former smoker and smokers & 29 & $5(17.2)$ & $4(13.8)$ & $1(3.4)$ \\
\hline$p$ value & & 0.55 & 0.029 & 0.36 \\
\hline \multicolumn{5}{|l|}{ Hypertension } \\
\hline No & 119 & $15(12.6)$ & $5(4.2)$ & $2(1.7)$ \\
\hline Yes & 27 & $5(18.5)$ & $2(7.4)$ & 0 \\
\hline$p$ value & & 0.53 & 0.61 & 1.0 \\
\hline \multicolumn{5}{|l|}{ Diabetes } \\
\hline No & 143 & 17 (11.9) & $7(4.9)$ & $2(1.4)$ \\
\hline Yes & 3 & $3(100.0)$ & 0 & 0 \\
\hline$p$ value & & 0.002 & 1.0 & 1.0 \\
\hline \multicolumn{5}{|l|}{ Previous breast RT } \\
\hline No & 117 & $10(8.5)$ & $6(5.1)$ & $1(0.8)$ \\
\hline Yes on ipsilateral side & 29 & $10(34.5)$ & $1(3.4)$ & $1(3.4)$ \\
\hline$p$ value & & 0.001 & 1.0 & 0.36 \\
\hline \multicolumn{5}{|l|}{ Previous breast surgery } \\
\hline No & 92 & $8(27.6)$ & $5(5.4)$ & 0 \\
\hline Ipsilateral & 38 & $12(31.6)$ & $2(5.3)$ & $2(5.3)$ \\
\hline Contralateral & 16 & 0 & 0 & 0 \\
\hline$p$ value & & 0.001 & 0.64 & 0.06 \\
\hline \multicolumn{5}{|l|}{ BMI $\left(\mathrm{kg} / \mathrm{m}^{2}\right)$} \\
\hline$<25$ & 114 & $10(8.8)$ & $5(4.4)$ & $2(1.7)$ \\
\hline$\geq 25$ & 32 & $10(31.2)$ & $2(6.2)$ & 0 \\
\hline$p$ value & & 0.003 & 0.65 & 1.0 \\
\hline \multicolumn{5}{|l|}{ Bilateral breast cancer } \\
\hline No & 140 & $20(14.3)$ & $7(5.0)$ & $2(1.4)$ \\
\hline Yes & 6 & 0 & 0 & 0 \\
\hline$p$ value & & 1.0 & 1.0 & 1.0 \\
\hline \multicolumn{5}{|l|}{ Mastectomy intent } \\
\hline Prophylactic & 29 & $3(10.3)$ & $1(3.4)$ & 0 \\
\hline Therapeutic & 117 & $17(14.5)$ & $6(5.1)$ & $2(1.7)$ \\
\hline$p$ value & & 0.77 & 1.0 & 1.0 \\
\hline \multicolumn{5}{|l|}{ Skin-reducing } \\
\hline No & 133 & $18(13.5)$ & $5(3.7)$ & $2(1.5)$ \\
\hline Yes & 13 & $2(15.4)$ & $2(15.4)$ & 0 \\
\hline$p$ value & & 0.69 & 0.12 & 1.0 \\
\hline \multicolumn{5}{|l|}{ Nipple-sparing } \\
\hline No & 28 & $3(10.7)$ & $1(3.6)$ & 0 \\
\hline Yes & 118 & $17(14.4)$ & $6(5.1)$ & $2(1.7)$ \\
\hline$p$ value & & 0.72 & 1.0 & 1.0 \\
\hline \multicolumn{5}{|l|}{ Mastectomy } \\
\hline Nipple/areola-sparing mastectomy & 110 & $16(14.5)$ & $5(4.5)$ & 2 \\
\hline Skin-reducing nipple-sparing mastectomy & 8 & $1(12.5)$ & $1(12.5)$ & 0 \\
\hline Skin-reducing mastectomy & 5 & $1(20.0)$ & $1(20.0)$ & 0 \\
\hline
\end{tabular}


Table 1 (continued)

\begin{tabular}{|c|c|c|c|c|}
\hline & $\begin{array}{l}\text { Cases } \\
n\end{array}$ & $\begin{array}{l}\text { Acute toxicity } \\
n(\%)\end{array}$ & $\begin{array}{l}\text { TE/I explant } \\
n(\%)\end{array}$ & $\begin{array}{l}\text { Late toxicity } \\
n(\%)\end{array}$ \\
\hline Skin-sparing mastectomy & 23 & $2(8.7)$ & 0 & 0 \\
\hline$p$ value & & 0.87 & 0.19 & 0.88 \\
\hline \multicolumn{5}{|l|}{ Axillary surgery } \\
\hline None & 34 & $7(20.6)$ & $1(2.9)$ & 0 \\
\hline SNB & 77 & $8(10.4)$ & $4(5.2)$ & 0 \\
\hline ALND & 35 & $5(14.3)$ & $2(5.7)$ & $2(5.7)$ \\
\hline$p$ value & & 0.35 & 0.84 & 0.04 \\
\hline \multicolumn{5}{|l|}{ Adjuvant RT } \\
\hline No & 108 & $14(13.0)$ & $4(3.7)$ & 0 \\
\hline Chest wall and regional node irradiation & 37 & $5(13.5)$ & $3(8.1)$ & $2(5.4)$ \\
\hline$p$ value & & 0.28 & 0.18 & 0.024 \\
\hline \multicolumn{5}{|l|}{ Chemotherapy* } \\
\hline None & 86 & $12(13.9)$ & $3(3.5)$ & $1(1.2)$ \\
\hline Postoperative & 43 & $5(11.6)$ & $1(2.3)$ & $1(2.3)$ \\
\hline Preoperative & 16 & $3(18.7)$ & $3(18.7)$ & 0 \\
\hline$p$ value & & 0.78 & 0.022 & 0.77 \\
\hline \multicolumn{5}{|l|}{ Adjuvant endocrine therapy* } \\
\hline No & 53 & $5(9.4)$ & $1(1.9)$ & $1(1.9)$ \\
\hline Yes & 92 & $15(16.3)$ & $6(6.5)$ & $1(1.1)$ \\
\hline$p$ value & & 0.32 & 0.42 & 1.0 \\
\hline \multicolumn{5}{|l|}{ (Neo)adjuvant trastuzumab* } \\
\hline No & 121 & $17(14.0)$ & $6(5.0)$ & $1(0.8)$ \\
\hline Yes & 24 & $3(12.5)$ & $1(4.2)$ & $1(4.2)$ \\
\hline$p$ value & & 1.0 & 1.0 & 0.31 \\
\hline \multicolumn{5}{|l|}{ Device } \\
\hline Tissue expander & 64 & $10(15.6)$ & $4(6.2)$ & 0 \\
\hline Implant & 82 & $10(12.2)$ & $3(3.7)$ & $2(2.4)$ \\
\hline$p$ value & & 0.63 & 0.70 & 0.50 \\
\hline Total & 146 & 20 & 7 & 2 \\
\hline
\end{tabular}

Association analysis by $\chi^{2}$ test between selected parameters and toxicity in 146 mastectomies and pre-pectoral IBBR. In bold $p$ value $<0.05$

$T E / I$ tissue expander/implant, $R T$ radiotherapy, $B M I$ body mass index, $S N B$ sentinel node biopsy, $A L N D$ axillary lymph node dissection

*Information not available in one case

to a mastectomy for cancer who required such a procedure for the contralateral side too, after a psychiatric evaluation. Nowadays a prophylactic mastectomy can be performed to BRCA-mutation carriers only after a genetic and psychooncologic counselling).

Most patients were aged more than 45 years. A minority of the series had received previous breast $\mathrm{RT}$ and previous breast surgery for BC.

Concerning postoperative treatments, 37 patients received PMRT, 92 endocrine treatments, and 43 chemotherapy. Main patient characteristics and association analysis by chi-square test between selected parameters and surgical outcomes (acute toxicity, TE/I explant, early late toxicity) are summarized in Table 1.
We recorded 20 representative acute complications, namely: 11 infections, 3 skin flap necroses, 3 wound dehiscences, 1 hematoma, 2 nipple necroses.

At univariate analysis, previous breast RT (34.5\% [RT] vs $8.5 \%$ [no RT]; $p=0.001$ ), previous ipsilateral breast surgery (31.6\% [previous surgery] vs 7.4\% [no previous surgery]; $p=0.001)$, and $\mathrm{BMI} \geq 25(31.3 \%[\geq 25]$ vs $8.8 \%[<25]$; $p=0.002)$ emerged as significant risk factors for acute toxicity $(n=20)$. At multivariate logistic analysis between individual parameters only $\mathrm{BMI} \geq 25$ (OR 4.44, 95\% CI $1.56-12.6 ; p=0.005)$ confirmed the statistical significance, while previous breast surgery on ipsilateral side was borderline (OR 3.74, 95\% CI 0.97-14.40; $p=0.055)$. Main results are described in Table 2. 
Table 2 Association analysis by logistic models between individual parameters and acute toxicity $(n=20)$ in 146 mastectomies and pre-pectoral IBBR

\begin{tabular}{|c|c|c|c|c|c|}
\hline & \multirow[t]{2}{*}{ Cases } & \multicolumn{2}{|l|}{ Univariate } & \multicolumn{2}{|l|}{ Multivariate } \\
\hline & & OR $(95 \% \mathrm{CI})$ & $p$ & OR $(95 \% \mathrm{CI})$ & $p$ \\
\hline \multicolumn{6}{|l|}{ Group age, years } \\
\hline$<45$ & 35 & 1 & & & \\
\hline $46-55$ & 64 & $0.74(0.22-2.52)$ & 0.63 & - & \\
\hline$>55$ & 47 & $1.23(0.37-4.15)$ & 0.74 & & \\
\hline \multicolumn{6}{|l|}{ Smoking habits } \\
\hline Never smoker & 117 & 1 & & - & \\
\hline Former smoker and smokers & 29 & $1.42(0.47-4.28)$ & 0.54 & & \\
\hline \multicolumn{6}{|l|}{ Hypertension } \\
\hline No & 119 & 1 & & - & \\
\hline Yes & 27 & $1.58(0.52-4.79)$ & 0.42 & & \\
\hline \multicolumn{6}{|l|}{ Diabetes } \\
\hline No & 143 & 1 & & - & \\
\hline Yes & 3 & Not evaluable & 0.99 & & \\
\hline \multicolumn{6}{|l|}{ Previous breast RT } \\
\hline No & 117 & 1 & & 1 & \\
\hline Yes on ipsilateral side & 29 & $5.63(2.07-15.4)$ & 0.001 & $2.22(0.56-8.74)$ & 0.26 \\
\hline \multicolumn{6}{|l|}{ Previous breast surgery } \\
\hline No previous surgery on IBBR side & 108 & 1 & & 1 & \\
\hline Previous surgery on ipsilateral side & 38 & $5.77(2.14-15.58)$ & 0.001 & $3.74(0.97-14.40)$ & 0.055 \\
\hline \multicolumn{6}{|l|}{ BMI $\left(\mathrm{kg} / \mathrm{m}^{2}\right)$} \\
\hline$<25$ & 114 & 1 & & 1 & \\
\hline$\geq 25$ & 32 & $4.73(1.76-12.7)$ & 0.002 & 4.67 (1.59-13.75) & $\mathbf{0 . 0 0 5}$ \\
\hline \multicolumn{6}{|l|}{ Bilateral breast cancer } \\
\hline No & 140 & 1 & & - & \\
\hline Yes & 6 & Not evaluable & 0.99 & & \\
\hline \multicolumn{6}{|l|}{ Mastectomy intent } \\
\hline Prophylactic & 29 & 1 & & - & \\
\hline Therapeutic & 117 & $1.47(0.40-5.41)$ & 0.56 & & \\
\hline \multicolumn{6}{|l|}{ Skin-reducing } \\
\hline No & 133 & 1 & & - & \\
\hline Yes & 13 & $1.16(0.24-5.68)$ & 0.85 & & \\
\hline \multicolumn{6}{|l|}{ Nipple-sparing } \\
\hline No & 28 & 1 & & & \\
\hline Yes & 118 & $1.40(0.38-5.16)$ & 0.61 & & \\
\hline \multicolumn{6}{|l|}{ Mastectomy } \\
\hline Nipple/areola-sparing mastectomy & 110 & 1 & & & \\
\hline Skin-reducing nipple-sparing mastectomy & 8 & $0.84(0.10-7.27)$ & 0.87 & - & \\
\hline Skin-reducing mastectomy & 5 & $1.47(0.15-14.0)$ & 0.74 & & \\
\hline Skin-sparing mastectomy & 23 & $0.56(0.12-2.62)$ & 0.46 & & \\
\hline \multicolumn{6}{|l|}{ Axillary surgery } \\
\hline None & 34 & 1 & & - & \\
\hline SNB plus ALND & 112 & $0.51(0.18-1.39)$ & 0.19 & & \\
\hline \multicolumn{6}{|l|}{ Postoperative RT } \\
\hline No & 108 & 1 & & - & \\
\hline Chest wall and regional node irradiation & 37 & $1.05(0.35-3.14)$ & 0.93 & & \\
\hline \multicolumn{6}{|l|}{ Chemotherapy* } \\
\hline None & 86 & 1 & & - & \\
\hline Postoperative plus preoperative & 59 & $0.97(0.37-2.53)$ & 0.95 & & \\
\hline
\end{tabular}


Table 2 (continued)

\begin{tabular}{|c|c|c|c|c|c|}
\hline & \multirow[t]{2}{*}{ Cases } & \multicolumn{2}{|l|}{ Univariate } & \multicolumn{2}{|l|}{ Multivariate } \\
\hline & & OR $(95 \% \mathrm{CI})$ & $p$ & OR $(95 \% \mathrm{CI})$ & $p$ \\
\hline No & 53 & 1 & & - & \\
\hline Yes & 92 & $1.87(0.64-5.48)$ & 0.25 & & \\
\hline \multicolumn{6}{|c|}{ (Neo)adjuvant trastuzumab* } \\
\hline No & 121 & 1 & & - & \\
\hline Yes & 24 & $0.87(0.24-3.25)$ & 0.84 & & \\
\hline \multicolumn{6}{|l|}{ Device } \\
\hline Tissue expander & 64 & 1 & & - & \\
\hline Implant & 82 & $0.75(0.29-1.93)$ & 0.55 & & \\
\hline
\end{tabular}

Univariate and multivariate logistic analysis: odds ratio (OR), 95\% CI and $p$ value. In bold $p$ value $<0.05$

$R T$ radiotherapy, $B M I$ body mass index, $S N B$ sentinel node biopsy, $A L N D$ axillary lymph node dissection

*Information not available in one case

Table 3 Association analysis by logistic models between individual parameters and tissue expander/implant (TE/I) explant $(n=7)$ in 146 mastectomies and pre-pectoral IBBR

\begin{tabular}{|c|c|c|c|c|c|}
\hline \multirow[t]{2}{*}{ Feature } & \multirow[t]{2}{*}{ Cases } & \multicolumn{2}{|l|}{ Univariate } & \multicolumn{2}{|l|}{ Multivariate } \\
\hline & & OR $(95 \% \mathrm{CI})$ & $p$ & OR $(95 \% \mathrm{CI})$ & $p$ \\
\hline \multicolumn{6}{|l|}{ Smoking habits } \\
\hline Never & 117 & 1 & & 1 & \\
\hline $\begin{array}{l}\text { Smokers or former } \\
\text { smokers }\end{array}$ & 29 & $6.08(1.28-28.88)$ & 0.023 & $7.32(1.37-39.08)$ & 0.02 \\
\hline \multicolumn{6}{|l|}{ Chemotherapy } \\
\hline None & 86 & 1 & & 1 & \\
\hline Postoperative & 43 & $0.66(0.07-6.53)$ & 0.72 & $0.69(0.07-7.14)$ & 0.76 \\
\hline Preoperative & 16 & $6.39(1.16-35.08)$ & 0.033 & $8.16(1.29-51.63)$ & 0.026 \\
\hline
\end{tabular}

Univariate and multivariate logistic analysis: odds ratio (OR), 95\% CI and $p$ value. In bold $p$ value $p<0.05$
At univariate analysis, significant risk-factor related to TE/I removal, 7 cases (reconstruction failure), were current or previous smoking exposition (13.8\% [smokers] vs $2.6 \%$ [non-smokers]; $p=0.023$ ) and preoperative chemotherapy $(18.8 \%$ [yes] vs $3.5 \%$ [no]; $p=0.033)$. At multivariate logistic analysis both features confirmed the statistical significance ( $p=0.02$ and $p=0.026$, respectively). The main results are reported in Table 3.

We recorded only two capsular grade III-IV contractures as late complication events at the 2-year follow up visit. No other cases of seroma, infection or different late complications were registered.

\section{Survival}

At a 4.0 year median follow up time (mean 3.9 years; range 3-5.5), 3 deaths, $5 \mathrm{LR}$, and $14 \mathrm{DM}$ occurred among the series. At time of analysis, OS was $78.1 \%$, LR freesurvival was $95.0 \%$, and DM free-survival was $71.6 \%$. Kaplan-Meier survival analysis for significant parameters are summarized in Table 4.

\section{Discussion}

Two-stage retro-pectoral implant-based BR has been the most common method for breast reconstruction for decades starting from the ' 80 s.

Lately, at the beginning of the new millennium, the introduction of matrixes, either synthetic or biologic, has opened the era of dual-plane techniques for an immediate implant positioning, the so called DTI. Eventually, in the last decade, recent advances in surgical techniques and matrixes technology have made pre-pectoral implant-based BR, either DTI or two-stage using TEs, feasible, showing enthusiastic results [28].

In a narrative review describing new insights on prepectoral BR [29], the authors showed that the subcutaneous pre-pectoral approach is safe, feasible, and has excellent short-term cosmetic and patient satisfaction outcomes. Even though further studies are strongly required to compare short- and long-term outcomes with the previous standard of care, early postoperative pain and quality of life evaluations seems to be equivalent between groups [30]. 
Table 4 Kaplan-Meier survival analysis in 117 patients treated with pre-pectoral IBBR at a 3-year median follow up time: patients at start, events (deaths, local recurrence, distant metastases), $p$ values from logrank test

\begin{tabular}{|c|c|c|c|c|}
\hline Variable & Cases & $\begin{array}{l}\text { Deaths } \\
n=3\end{array}$ & $\begin{array}{l}\text { Local recurrence } \\
n=5\end{array}$ & $\begin{array}{l}\text { Distant metastases } \\
n=14\end{array}$ \\
\hline \multicolumn{5}{|l|}{ Group age, years } \\
\hline$<45$ & 23 & $2(8.7)$ & $2(8.7)$ & $2(8.7)$ \\
\hline $46-55$ & 53 & 0 & $2(3.8)$ & $7(13.2)$ \\
\hline$>55$ & 41 & $1(2.4)$ & $1(2.4)$ & $5(12.2)$ \\
\hline$p$ value & & 0.10 & 0.46 & 0.73 \\
\hline \multicolumn{5}{|l|}{ Smoking habits } \\
\hline Never smoker & 95 & $2(2.1)$ & $4(4.2)$ & $13(13.7)$ \\
\hline Former smoker and smokers & 22 & $1(4.5)$ & $1(4.5)$ & $1(4.5)$ \\
\hline$p$ value & & 0.96 & 0.90 & 0.29 \\
\hline \multicolumn{5}{|l|}{ Hypertension } \\
\hline No & 92 & $2(2.2)$ & $5(5.4)$ & $9(9.8)$ \\
\hline Yes & 25 & $1(4.0)$ & 0 & $5(20)$ \\
\hline$p$ value & & 0.47 & 0.28 & 0.064 \\
\hline \multicolumn{5}{|l|}{ BRCA mutation-carrier } \\
\hline No & 111 & $3(2.7)$ & $5(4.5)$ & $13(11.7)$ \\
\hline Yes & 6 & 0 & 0 & $1(16.7)$ \\
\hline$p$ value & & 0.82 & 0.61 & 0.66 \\
\hline \multicolumn{5}{|l|}{ Diabetes } \\
\hline No & 114 & $3(2.6)$ & $5(4.4)$ & $14(12.3)$ \\
\hline Yes & 3 & 0 & 0 & 0 \\
\hline$p$ value & & 0.87 & 0.72 & 0.57 \\
\hline \multicolumn{5}{|l|}{ Previous breast RT } \\
\hline No & 97 & $3(3.1)$ & $4(4.1)$ & $12(12.4)$ \\
\hline Yes on ipsilateral side & 20 & 0 & $1(5.0)$ & $2(10.0)$ \\
\hline$p$ value & & 0.44 & 0.91 & 0.68 \\
\hline \multicolumn{5}{|l|}{ Previous breast surgery } \\
\hline No & 78 & $2(2.6)$ & $3(3.8)$ & $10(12.8)$ \\
\hline Ipsilateral & 33 & 0 & $1(3.0)$ & $3(9.1)$ \\
\hline Contralateral & 6 & $1(16.7)$ & $1(16.7)$ & $1(16.7)$ \\
\hline$p$ value & & 0.22 & 0.26 & 0.80 \\
\hline \multicolumn{5}{|l|}{ BMI $\left(\mathrm{kg} / \mathrm{m}^{2}\right)$} \\
\hline$<25$ & 90 & $2(2.2)$ & $5(5.5)$ & $11(12.2)$ \\
\hline$\geq 25$ & 27 & $1(3.7)$ & 0 & $3(11.1)$ \\
\hline$p$ value & & 0.50 & 0.22 & 0.95 \\
\hline \multicolumn{5}{|l|}{ Bilateral breast cancer } \\
\hline No & 111 & $3(2.7)$ & $5(4.5)$ & $14(12.6)$ \\
\hline Yes & 6 & 0 & 0 & 0 \\
\hline$p$ value & & 0.82 & 0.59 & 0.34 \\
\hline \multicolumn{5}{|l|}{ Mastectomy } \\
\hline Nipple/areola-sparing mastectomy & 84 & $2(2.4)$ & $4(4.8)$ & $7(8.3)$ \\
\hline Skin-reducing nipple-sparing mastectomy & 5 & 0 & $1(20.0)$ & 0 \\
\hline Skin-reducing mastectomy & 5 & 0 & 0 & $1(20.0)$ \\
\hline Skin-sparing mastectomy & 23 & $1(4.3)$ & 0 & $6(26.1)$ \\
\hline$p$ value & & 0.75 & 0.12 & 0.21 \\
\hline \multicolumn{5}{|l|}{ Axillary surgery } \\
\hline None & 19 & 0 & $1(5.3)$ & $2(10.5)$ \\
\hline SNB & 63 & $1(1.6)$ & $2(3.2)$ & $2(3.2)$ \\
\hline ALND & 35 & $2(5.7)$ & $2(5.7)$ & $10(28.6)$ \\
\hline$p$ value & & 0.85 & 0.84 & 0.008 \\
\hline
\end{tabular}


Table 4 (continued)

\begin{tabular}{lllll}
\hline Variable & Cases & $\begin{array}{l}\text { Deaths } \\
n=3\end{array}$ & $\begin{array}{l}\text { Local recurrence } \\
n=5\end{array}$ & $\begin{array}{l}\text { Distant metastases } \\
n=14\end{array}$ \\
\hline No & 83 & $1(1.2)$ & $3(3.6)$ & $6(7.2)$ \\
Chest wall and regional node irradiation & 33 & $2(6.1)$ & $2(6.1)$ & $8(24.2)$ \\
$p$ value & & 0.57 & 0.74 & $\mathbf{0 . 0 3 6}$ \\
Chemotherapy* & & & & \\
None & 65 & 0 & $1(1.5)$ & $1(1.5)$ \\
Postoperative & 40 & $1(2.5)$ & $3(7.5)$ & $8(20.0)$ \\
Preoperative & 11 & $2(18.2)$ & $1(9.1)$ & $5(45.4)$ \\
$p$ value & & 0.11 & 0.24 & $\mathbf{0 . 0 0 0 1}$ \\
Adjuvant endocrine therapy* & & & & $5(14.7)$ \\
No & 34 & 0 & $2(5.9)$ & $9(11.0)$ \\
Yes & 82 & $3(3.6)$ & $3(3.6)$ & 0.59 \\
$p$ value & & 0.23 & 0.59 & $11(11.8)$ \\
(Neo)adjuvant trastuzumab* & & & & $3(13.0)$ \\
No & 93 & $3(3.2)$ & $4(4.3)$ & 0.90 \\
Yes & 23 & 0 & $1(4.3)$ & $14(12.0)$ \\
$p$ value & & 0.36 & 0.95 &
\end{tabular}

In bold $p$ value $<0.05$

$R T$ radiotherapy, $B M I$ body mass index, $S N B$ sentinel node biopsy, $A L N D$ axillary lymph node dissection

*Information not available in one case

In the last 5 years the pre-pectoral approach has reached a sky-rocketing success in the breast reconstruction scenario, and, even in the absence of any level IA evidence, many selection criteria and algorithm have been proposed. Nonetheless, a cloudy area of surgical management entails the relationship between pre-pec IBBR and radiation therapy, either as a previous treatment or as a PMRT treatment, with a scarce presence in the literature. We decided to go back to our very early cases, at the very beginning of the pre-pec era, which we started in 2011, when selection criteria were quite loose. Analyzing an early population we have had the opportunity to include many different characteristics and risk factors in a quite large series, with also a significant number of cases with previous breast RT and with PMRT, to perform a multivariate analysis with many unselected patients and draw quite comprehensive conclusions.

We confirmed the overall safety of the pre-pectoral approach, showing $13.7 \%$ of acute postoperative complications with $4.8 \% \mathrm{TE} / \mathrm{I}$ failure rate, and $1.4 \%$ late surgical complications at the 2-year assessment.

An increasing number of women who undergo IBBR either one or two-stage will require PMRT [31], since the meta-analysis of Early Breast Cancer Trialists' Collaborative Group (EBCTCG) confirmed a significant reduction of both LR and BC mortality in patients with pathological nodal involvement [32]. Moreover, regional nodal irradiation improved outcomes for most of high-intermediate risk BC patients [33, 34].
Concerning the role of adjuvant treatments great attention focused on PMRT over time, and the impact of its timing to a TE or a permanent implant has not been clarified yet. Patients receiving PMRT after pre-pectoral BR showed a capsular contracture rate and severity significantly lower as compared to those receiving PMRT after retro-pectoral breast reconstruction [35-37].

Sinnott and colleagues [35] in a recently published study on 274 patients showed a capsular contracture rate difference of $36.1 \%(52.2 \%$ vs $16.1 \%$; $p=0.0018)$ and a $61.1 \%$ difference of Baker grades 3 or 4 severity $(83.3 \%$ vs $22.2 \%$; $p=0.0092$ ) in favor of pre-pectoral BR. Ricci and colleagues [36] published a review including 20 studies (range 2000-2016) and 2348 patients showing that PMRT delivered on TE resulted in higher rates of reconstructive failure as compared to PMRT applied to permanent implants (20\% vs $13.4 \%$, OR $2.33 ; p=0.0083$ ), but lower rates of capsular contracture ( $24.5 \%$ vs $49.4 \%$, OR $0.53 ; p=0.083)$.

Although the risk of reconstructive failure is significantly higher for patients with TE radiation compared to patients with permanent implant radiation, the aesthetic results and capsular contracture rates are slightly better [37]. Therefore, immediate TE/I reconstruction seems to be a reasonable surgical option also in the setting of PMRT [31].

In our study 37 cases received PMRT (25.3\%), 19 irradiated the TE and 18 the permanent implant. At the 2-year late complication assessment only two patients showed grade IIIIV capsular contracture (5.4\% of patients receiving PMRT), 
both in case of immediate permanent implant reconstruction. The lower rate of capsular contracture in TE IBBR pre-pec cases submitted to PMRT could be explained, in our experience, to the fact that a fat graft procedure on the irradiated skin flap is always performed during the second stage procedure in such cases, thus permitting a regenerative process to the irradiated tissues. Moreover, thanks to the recent advance in surgical and radiation techniques [38], PMRT seemed not to be the only risk factor for unsatisfactory postoperative outcomes in pre-pectoral reconstructions.

Indeed, in our single-center experience, we evidenced $\mathrm{BMI} \geq 25$ at diagnosis (OR 4.44, CI 95\% 1.56-12.6; $p=0.005)$ as an independent risk-factor of acute surgical complications development. Previous breast surgery on the IBBR side was almost close to significance (OR 3.74, 95\% CI $0.97-14.40 ; p=0.055)$. Smoking habits (OR 7.32, CI $95 \% 1.37-39.08 ; p=0.02)$ and preoperative chemotherapy (OR 8.16, CI 95\% 1.29-51.6; $p=0.026$ ) resulted as independent risk-factors of TE/I explant (reconstruction failure).

Therefore, patient selection for the pre-pectoral technique seems to be crucial and it is dependent on patient-related factors (such as smoking, comorbidities, previous treatments such as RT, breast size, BMI, and lifestyle), as well as adjuvant prescribed treatments (local and systemic therapies) [29, 39-43].

Concerning survival outcomes, a direct comparison between published studies is limited mainly due to different reconstruction techniques used, several missing data on RT (schedule, volume, quality assurance) and systemic therapies (drugs, regimens, doses, setting) [44-48]. However, in a meta-analysis on 19 studies immediate breast reconstruction after mastectomy showed at least equivalent overall survival and disease-free survival as compared to mastectomy alone [49].

Although the short-term follow up did not allowed any definitive conclusions on survival, not surprisingly our study identified a more extensive local treatment (i.e., PMRT, ALND) and systemic treatments needs (i.e., preoperative chemotherapy) as independent risk factors for DM development.

\section{Conclusions}

In our experience, pre-pectoral IBBR, either one or twostage, was safe independent of device (TE or implant) and postoperative treatments. Preoperative information should be carefully considered to assess the overall procedural risks and to individualize the reconstructive management, particularly BMI and previous ipsilateral breast treatments. On the other hand, a foreseen PMRT doesn't seem to be a possible hindrance to the pre-pec IBBR. However, further investigations and mature follow-up are warranted to confirm these results.

Acknowledgements We would like to thank Matteo Mariotti, Camilla Delli Paoli, Emanuela Olmetto, and Virginia Maragna involved in data collection (University of Florence, Florence, Italy). Partial data were presented at 2018 San Antonio Breast Cancer Symposium.

Author contributions Conceptualization: MB, IM; methodology: MB, IM, CB; formal analysis and investigation: CS, CB, VS, LV, SS, DB; writing and original draft preparation: $\mathrm{MB}, \mathrm{CB}$; writing, review and editing: IM, ID, LV, GS, CB, VL; resources: SS, VL, GS, VS and supervision: LL, JN, LO, SB.

Funding None.

\section{Declarations}

Conflict of interest All authors have no conflict of interest to declare.

Ethical approval Informed consent was obtained from all individual participants included in the study.

\section{References}

1. American Society of Plastic Surgeons Statistics. http://www.plast icsurgery.org/Documents/news-resources/statistics. Accessed 4 Feb 2016.

2. Endara M, Chen D, Verma K, Nahabedian MY, Spear SL. Breast reconstruction following nipple-sparing mastectomy: a systematic review of the literature with pooled analysis. Plast Reconstr Surg. 2013;132(5):1043-54.

3. Snyderman RK, Guthrie RH. Reconstruction of the female breast following radical mastectomy. Plast Reconstr Surg. 1971;47:565e7.

4. Radovan C. Breast reconstruction after mastectomy using the temporary expander. Plast Reconstr Surg. 1982;69(2):195-208.

5. Lapin R, Elliott M, Juri H. The use of an integral tissue expander for primary breast reconstruction. Aesthet Plast Surg. 1985;9:221e6.

6. Gruber RP, Kahn RA, Lash H, Maser MR, Apfelberg DB, Laub DR. Breast reconstruction following mastectomy: a comparison of submuscular and subcutaneous techniques. Plast Reconstr Surg. 1981;67:312e7.

7. Argenta LC, Marks MW, Grabb WC. Selective use of serial expansion in breast reconstruction. Ann Plast Surg. 1983;11(3):188-95.

8. Nahabedian MY. Acellular dermal matrices in primary breast reconstruction: principles, concepts and indications. Plast Reconstr Surg. 2012;130:44-53.

9. Casella D, Bernini M, Bencini L, et al. TiLoop ${ }^{\circledR}$ Bra mesh used for immediate breast reconstruction: comparison of retropectoral and subcutaneous implant placement in a prospective singleinstitution series. Eur J Plast Surg. 2014;37(11):599-604.

10. Reitsamer R, Peintinger F. Prepectoral implant placement and complete coverage with porcine acellular dermal matrix: a new technique for direct-to-implant breast reconstruction after nipple-sparing mastectomy. J Plast Reconstr Aesthet Surg. 2015;68(2):162-7.

11. Woo A, Harless C, Jacobson SR. Revisiting an old place: single surgeon experience on post-mastectomy subcutaneous implant 
based breast reconstruction. Plast Reconstr Surg. 2015;136(4 Suppl):83.

12. Bernini M, Calabrese C, Cecconi L, et al. Subcutaneous directto-implant breast reconstruction: surgical, functional, and aesthetic results after long-term follow-up. Plast Reconstr Surg Glob Open. 2016;3(12):e574.

13. Casella D, Calabrese C, Bianchi S, et al. Subcutaneous tissue expander placement with synthetic titanium-coated mesh in breast reconstruction: long-term results. Plast Reconstr Surg Glob Open. 2016;3(12):e577.

14. Zhu L, Mohan AT, Abdelsattar JM, et al. Comparison of subcutaneous versus submuscular expander placement in the first stage of immediate breast reconstruction. J Plast Reconstr Aesthet Surg. 2016;69(4):e77-86.

15. Sigalove S, Maxwell GP, Sigalove NM, et al. Prepectoral implant-based breast reconstruction: rationale, indications, and preliminary results. Plast Reconstr Surg. 2017;139(2):287-94.

16. Bettinger LN, Waters LM, Reese SW, et al. Comparative study of prepectoral and subpectoral expander-based breast reconstruction and Clavien IIIb score outcomes. Plast Reconstr Surg Glob Open. 2017;5(7):e1433.

17. Nadeem R. Prepectoral implant-based breast reconstruction; complete acellular dermal matrix wrap or anterior circumferential cover. Breast J. 2018;24(2):223-4.

18. Vidya R. Prepectoral breast reconstruction or muscle-sparing technique with the Braxon porcine acellular dermal matrix. Plast Reconstr Surg Glob Open. 2017;5(6):e1364.

19. Sbitany H, Piper M, Lentz R. Prepectoral breast reconstruction: a safe alternative to submuscular prosthetic reconstruction following nipple-sparing mastectomy. Plast Reconstr Surg. 2017;140(3):432-43.

20. Vidya R, Masià J, Cawthorn S, et al. Evaluation of the effectiveness of the prepectoral breast reconstruction with Braxon dermal matrix: first multicenter European report on 100 cases. Breast J. 2017;23(6):670-6

21. Jafferbhoy S, Chandarana M, Houlihan M, et al. Early multicentre experience of pre-pectoral implant based immediate breast reconstruction using Braxon®. Gland Surg. 2017;6(6):682-8.

22. Tasoulis MK, Iqbal FM, Cawthorn S, MacNeill F, Vidya R. Subcutaneous implant breast reconstruction: time to reconsider? Eur J Surg Oncol. 2017;43(9):1636-46.

23. Chatterjee A, Nahabedian MY, Gabriel A, et al. Early assessment of post-surgical outcomes with pre-pectoral breast reconstruction: a literature review and meta-analysis. J Surg Oncol. 2018;117(6):1119-30.

24. Wazir U, Mokbel K. The evolving role of pre-pectoral ADMassisted implant-based immediate breast reconstruction following skin-sparing mastectomy. Am J Surg. 2018;216(3):639-40.

25. Mitchell MP, Wagner J, Butterworth J. Subcutaneous implantbased breast reconstruction, a modern challenge in postmastectomy radiation planning. Pract Radiat Oncol. 2018;8(3):153-6.

26. Harvey KL, Mills N, White P, Holcombe C, Potter S, Pre-BRA Feasibility Study Steering Group. The Pre-BRA (pre-pectoral Breast Reconstruction EvAluation) feasibility study: protocol for a mixed-methods IDEAL $2 \mathrm{a} / 2 \mathrm{~b}$ prospective cohort study to determine the safety and effectiveness of pre-pectoral implantbased breast reconstruction. BMJ Open. 2020;10(1):e03364. https://doi.org/10.1136/bmjopen-2019-033641.

27. Baker JL Jr. Owsley JQ Jr, Peterson RA, eds. Augmentation mammaplasty. In: Symposium on aesthetic surgery of the breast. 1978:St. Louis: Mosby;256-263.

28. Elswick SM, Harless CA, Bishop SN, et al. Prepectoral implantbased breast reconstruction with postmastectomy radiation therapy. Plast Reconstr Surg. 2018;142(1):1-12.
29. Vidya R, Iqbal FM. A guide to prepectoral breast reconstruction: a new dimension to implant-based breast reconstruction. Clin Breast Cancer. 2017;17(4):266-71.

30. Baker BG, Irri R, MacCallum V, Chattopadhyay R, Murphy J, Harvey JR. A prospective comparison of short-term outcomes of subpectoral and prepectoral strattice-based immediate breast reconstruction. Plast Reconstr Surg. 2018;141(5):1077-84.

31. Ho AL, Bovill ES, Macadam SA, Tyldesley S, Giang J, Lennox PA. Postmastectomy radiation therapy after immediate twostage tissue expander/implant breast reconstruction: a University of British Columbia perspective. Plast Reconstr Surg. 2014;134(1):1e-10e.

32. Early Breast Cancer Trialists' Collaborative Group (EBCTCG), Darby S, McGale P, et al. Effect of radiotherapy after breastconserving surgery on 10-year recurrence and 15-year breast cancer death: meta-analysis of individual patient data for 10,801 women in 17 randomised trials. Lancet. 2011;378(9804):1707-16.

33. Whelan TJ, Olivotto IA, MA.20 Study Investigators, et al. Regional nodal irradiation in early-stage breast cancer. N Engl J Med. 2015;373(4):307-16. https://doi.org/10.1056/NEJMoa1415 340.

34. Poortmans PM, Collette S, EORTC Radiation Oncology and Breast Cancer Groups, et al. Internal mammary and medial supraclavicular irradiation in breast cancer. N Engl J Med. 2015;373(4):317-27. https://doi.org/10.1056/NEJMoa1415369.

35. Sinnott CJ, Persing SM, Pronovost M, Hodyl C, McConnell D, Ott YA. Impact of postmastectomy radiation therapy in prepectoral versus subpectoral implant-based breast reconstruction. Ann Surg Oncol. 2018;25(10):2899-908.

36. Ricci JA, Epstein S, Momoh AO, Lin SJ, Singhal D, Lee BT. A meta-analysis of implant-based breast reconstruction and timing of adjuvant radiation therapy. J Surg Res. 2017;218:108-16.

37. Cordeiro PG, Albornoz CR, McCormick B, Hudis CA, Hu Q, Heerdt A, Matros E. What is the optimum timing of postmastectomy radiotherapy in two-stage prosthetic reconstruction: radiation to the tissue expander or permanent implant? Plast Reconstr Surg. 2015;135(6):1509-17.

38. Koutcher L, Ballangrud A, Cordeiro PG, et al. Postmastectomy intensity modulated radiation therapy following immediate expander-implant reconstruction. Radiother Oncol. 2010;94(3):319-23.

39. Sbitany H. Important considerations for performing prepectoral breast reconstruction. Plast Reconstr Surg. 2017;140:7S-13S.

40. Ito $\mathrm{H}$, Ueno $\mathrm{T}$, Suga $\mathrm{H}$, et al. Risk factors for skin flap necrosis in breast cancer patients treated with mastectomy followed by immediate breast reconstruction. World J Surg. 2018. https://doi. org/10.1007/s00268-018-4852-y.

41. Riggio E, Toffoli E, Tartaglione C, Marano G, Biganzoli E. Local safety of immediate reconstruction during primary treatment of breast cancer. Direct-to-implant versus expander-based surgery. J Plast Reconstr Aesthet Surg. 2018; pii: S1748-6815(18)30368-1.

42. Lam TC, Borotkanics R, Hsieh F, Salinas J, Boyages J. Immediate two-stage prosthetic breast reconstruction failure: radiation is not the only culprit. Plast Reconstr Surg. 2018;141(6):1315-24.

43. McCarthy CM, Mehrara BJ, Riedel E, et al. Predicting complications following expander/implant breast reconstruction: an outcomes analysis based on preoperative clinical risk. Plast Reconstr Surg. 2008;121(6):1886-92.

44. Bezuhly M, Temple C, Sigurdson LJ, et al. Immediate postmastectomy reconstruction is associated with improved breast cancer-specific survival: evidence and new challenges from the Surveillance, Epidemiology, and End Results database. Cancer. 2009; 115:4648-54.

45. Lee HH, Hou MF, Wei SY, et al. Comparison of long-term outcomes of postmastectomy radiotherapy between breast cancer 
patients with and without immediate flap reconstruction. PLoS ONE. 2016;11:e0148318.

46. McCarthy CM, Pusic AL, Sclafani L, et al. Breast cancer recurrence following prosthetic, postmastectomy reconstruction: incidence, detection, and treatment. Plast Reconstr Surg. 2008;121:381-8.

47. Aurilio G, Bagnardi V, Nole F, et al. Outcome of immediate breast reconstruction in patients with nonendocrine-responsive breast cancer: a monoinstitutional case-control study. Clin Breast Cancer. 2015;15:e237-241.

48. Eriksen C, Frisell J, Wickman M, et al. Immediate reconstruction with implants in women with invasive breast cancer does not affect oncological safety in a matched cohort study. Breast Cancer Res Treat. 2011;127:439-46.

49. Zhang P, Li CZ, Wu CT, et al. Comparison of immediate breast reconstruction after mastectomy and mastectomy alone for breast cancer: A meta-analysis. Eur J Surg Oncol. 2017;43:285-93.

Publisher's Note Springer Nature remains neutral with regard to jurisdictional claims in published maps and institutional affiliations. 